\title{
Sequential closedness of Boolean algebras of projections in Banach spaces
}

\author{
by \\ D. H. Fremlin (Colchester), B. De Pagter (Delft) \\ and W. J. RICKER (Eichstätt)
}

\begin{abstract}
Complete and $\sigma$-complete Boolean algebras of projections acting in a Banach space were introduced by W. Bade in the 1950's. A basic fact is that every complete Boolean algebra of projections is necessarily a closed set for the strong operator topology. Here we address the analogous question for $\sigma$-complete Boolean algebras: are they always a sequentially closed set for the strong operator topology? For the atomic case the answer is shown to be affirmative. For the general case, we develop criteria which characterize when a $\sigma$-complete Boolean algebra of projections is sequentially closed. These criteria are used to show that both possibilities occur: there exist examples which are sequentially closed and others which are not (even in Hilbert space).
\end{abstract}

1. Introduction. In the Banach space setting, $\sigma$-complete and complete Boolean algebras (briefly, B.a.'s) of projection operators were intensively studied by W. Bade ([1], [2]). Such objects are a natural extension of the fundamental notion of the resolution of the identity of a normal operator in Hilbert space. The definition of such $(\sigma-)$ complete B.a.'s of projections (see Section 2) is directly connected to the (non-metrizable) strong operator topology. Accordingly, the theory of such B.a.'s is somewhat different to the theory of abstract B.a.'s.

It was already noted from the outset of the theory that there are subtle topological and order-theoretic distinctions between the notions of $\sigma$-completeness and completeness. For instance, let $X$ denote the Hilbert space $\ell^{2}([0,1])$ and $\Sigma$ the family of all Borel subsets of $[0,1]$. For each $E \in \Sigma$ define a self-adjoint projection $P(E)$ by $P(E) x=\chi_{E} x$ for each $x \in X$. Then $P(E) \in \mathcal{L}(X)$, where $\mathcal{L}(X)$ denotes the space of all bounded linear operators on (any Banach space) $X$, and $\mathcal{M}=P(\Sigma) \subseteq \mathcal{L}(X)$ is a $\sigma$-complete B.a. of projections which fails to be complete. For a general $\sigma$-complete B.a. of pro-

2000 Mathematics Subject Classification: Primary 46E27, 47B40; Secondary 46B26, $46 \mathrm{~B} 42$.

Key words and phrases: Boolean algebras of projections, sequential closedness, Bade functionals, band projections, spaces of measures. 
jections $\mathcal{M}$ in a Banach space $X$, the distinction is somewhat delicate. For, if $Y$ is the closed subspace of $X$ generated by $\left\{B x_{n}: B \in \mathcal{M}, n \in \mathbb{N}\right\}$, then the restrictions $\left.\mathcal{M}\right|_{Y}=\left\{\left.B\right|_{Y}: B \in \mathcal{M}\right\}$ form a complete B.a. of projections in $Y$ ([6, XVII, Lemma 3.21]). In particular, whenever $X$ is separable the notions of $\sigma$-complete and complete coincide.

It is known that every complete B.a. of projections $\mathcal{M} \subseteq \mathcal{L}(X)$ is necessarily a $\tau_{\mathrm{s}}$-closed set for the strong operator topology $\tau_{\mathrm{s}}$ in $\mathcal{L}(X)$ ([6, XVII, Corollary 3.7]). This raises the following

QUESTION. Is every $\sigma$-complete B.a. of projections in a Banach space $X$ necessarily a sequentially $\tau_{\mathrm{s}}$-closed subset of $\mathcal{L}(X)$ ?

An equivalent formulation of this question appears in 323Z (Problem) of [8]. The aim of this paper is to give a complete answer to this question.

The above example given in $X=\ell^{2}([0,1])$ is immediately relevant. The B.a. $\mathcal{M}$ given there fails to be $\tau_{\mathrm{s}}$-closed in $\mathcal{L}(X)$, but it is sequentially $\tau_{\mathrm{s}^{-}}$ closed. That is, if $\left\{P_{n}\right\}_{n=1}^{\infty} \subseteq \mathcal{M}$ is any $\tau_{\mathrm{s}}$-convergent sequence, with limit $Q \in \mathcal{L}(X)$ say, then actually $Q \in \mathcal{M}$. This is no accident. It is shown in [14, p. 367] that whenever $\mathcal{M} \subseteq \mathcal{L}(X)$ is Bade atomic and $\sigma$-complete, then it is always sequentially $\tau_{\mathrm{s}}$-closed in $\mathcal{L}(X)$. Here Bade atomic means that there is a family of atoms $\left\{P_{\alpha}\right\}_{\alpha \in \mathcal{A}}$ in $\mathcal{M}$ which generate $\mathcal{M}$ in the sense that, for every $0 \neq P \in \mathcal{M}$, there exists a non-empty subset $\mathcal{B} \subseteq \mathcal{A}$ with $P=\sum_{\alpha \in \mathcal{B}} P_{\alpha} ;$ summability of the series is meant as the $\tau_{\mathrm{s}}$-limit of the net of partial sums over all finite subsets of $\mathcal{B}$. The above-mentioned example in $\ell^{2}([0,1])$ is clearly Bade atomic in this sense. In Section 2 we will show that the above-mentioned result concerning Bade atomic B.a.'s of projections can actually be further extended to include arbitrary atomic, $\sigma$-complete B.a.'s of projections, where atomic is meant purely in the sense of abstract Boolean algebras. By way of examples it is shown that this extension is genuine. So, the atomic case is now completely understood.

Section 3 is concerned with presenting criteria which characterize precisely whether or not a given $\sigma$-complete B.a. $\mathcal{M}$ is sequentially $\tau_{\mathrm{s}}$-closed in $\mathcal{L}(X)$. The idea is to represent $\mathcal{M}$ as the range of an $\mathcal{L}(X)$-valued spectral measure defined on some $\sigma$-algebra of sets $\Sigma$, and then to consider certain families $\Phi$ of non-negative measures on $\Sigma$ induced by so-called Bade functionals ([6, XVII, Lemma 3.12]). The criteria presented are based on the notion of sequential $\Phi$-completeness of the $\sigma$-algebra $\Sigma$ (see Section 3 for the definition).

The most interesting part of the paper, perhaps, is the final Section 4, which is concerned with examples. It turns out that to exhibit measurable spaces $(\Omega, \Sigma)$ and families of non-negative measures $\Phi$ for which $\Sigma$ is not sequentially $\Phi$-complete and then, based on the properties of $\Phi$, to construct non-atomic, $\sigma$-complete B.a.'s of projections in some appropriate 
non-separable Banach space, is rather non-trivial. Briefly, the answer to the above Question turns out to be no, in general. There exist examples of nonatomic, $\sigma$-complete B.a.'s of projections $\mathcal{M} \subseteq \mathcal{L}(X)$ which are sequentially $\tau_{\mathrm{s}}$-closed but not $\tau_{\mathrm{s}}$-closed and others which fail to be sequentially $\tau_{\mathrm{s}}$-closed (even in a Hilbert space!). In the latter case, we point out that the sequential closure of $\mathcal{M}$, formed in the non-metrizable space $\mathcal{L}_{\mathrm{s}}(X)=\left(\mathcal{L}(X), \tau_{\mathrm{s}}\right)$, is always a $\sigma$-complete B.a. of projections ([13, Corollary 2.1]). Finally, we remark that there seems to be a distinct lack of concrete examples of nonatomic, $\sigma$-complete but not complete B.a.'s of projections in Banach spaces available. In particular, such B.a.'s should not admit any separating vector ([11, Lemma 1]). Hopefully, the examples of Section 4 go some way towards rectifying this situation.

2. Atomic Boolean algebras. Let $X$ be a Banach space. All B.a.'s of projections $\mathcal{M} \subseteq \mathcal{L}(X)$ are assumed to have the identity operator $I$ on $X$ as their unit. We say that $\mathcal{M}$ is complete (resp. $\sigma$-complete) in the sense of Bade if it is complete (resp. $\sigma$-complete) as an abstract B.a. and if, for every family (resp. countable family) $\mathcal{D} \subseteq \mathcal{M}$, we have

and

$$
\left(\bigwedge_{D \in \mathcal{D}} D\right)(X)=\bigcap_{D \in \mathcal{D}} D(X)
$$

$$
\left(\bigvee_{D \in \mathcal{D}} D\right)(X)=\overline{\mathrm{sp}}\left\{\bigcup_{D \in \mathcal{D}} D(X)\right\}
$$

the closed subspace of $X$ generated by $\bigcup_{D \in \mathcal{D}} D(X)$; see [1] or [6, Chapter XVII], for example.

Let $\mathcal{M} \subseteq \mathcal{L}(X)$ be a B.a. of projections. A non-zero element $P \in \mathcal{M}$ is called an atom if, whenever $Q \in \mathcal{M}$ satisfies $Q \leq P$, then either $Q=0$ or $Q=P$. We say that $\mathcal{M}$ is atomic if for every $0 \neq Q \in \mathcal{M}$ there exists an atom $P \leq Q$ (see e.g. [16, Section 9]). Before proving the main result of this section, we first clarify the relation between atomic and Bade atomic B.a.'s.

Lemma 2.1. Let $X$ be a Banach space and $\mathcal{M} \subseteq \mathcal{L}(X)$ be a B.a. of projections.

(i) If $\mathcal{M}$ is Bade atomic, then it is atomic.

(ii) If $\mathcal{M}$ is Bade complete, then $\mathcal{M}$ is Bade atomic if and only if it is atomic.

Proof. Statement (i) follows immediately from the definition of Bade atomic (see Section 1). For the proof of (ii) it suffices to show that if $\mathcal{M}$ is atomic and Bade complete, then $\mathcal{M}$ is Bade atomic. To this end, let $\left\{P_{\alpha}: \alpha \in \mathcal{A}\right\}$ be the collection of all atoms in $\mathcal{M}$. Given $0 \neq Q \in \mathcal{M}$, let $\mathcal{B}=\left\{\alpha \in \mathcal{A}: P_{\alpha} \leq Q\right\}$. It is clear that $Q=\bigvee_{\alpha \in \mathcal{B}} P_{\alpha}$. Denoting by 
$\mathcal{F}$ the collection of all finite subsets of $\mathcal{B}$ and defining $P_{F}=\sum_{\alpha \in F} P_{\alpha}$ for all $F \in \mathcal{F}$, it is clear that $P_{F} \uparrow_{\mathcal{F}} Q$ in $\mathcal{M}$. Since $\mathcal{M}$ is Bade complete, we may conclude by Lemma XVII.3.4 in [6] that $Q=\lim _{F \in \mathcal{F}} P_{F}$ in $\mathcal{L}_{\mathrm{s}}(X)$, i.e., $Q=\sum_{\alpha \in \mathcal{B}} P_{\alpha}$ as a $\tau_{\mathrm{s}}$-summable series.

Theorem 2.2. Let $X$ be a Banach space. Then every atomic, Bade $\sigma$ complete B.a. of projections in $X$ is sequentially closed in $\mathcal{L}_{\mathrm{s}}(X)$.

Proof. Let $\mathcal{M} \subseteq \mathcal{L}(X)$ be a Bade $\sigma$-complete, atomic B.a. and suppose that $\left\{H_{n}\right\}_{n=1}^{\infty} \subseteq \mathcal{M}$ is a sequence satisfying $H_{n} \rightarrow H$ in $\mathcal{L}_{\mathrm{s}}(X)$ for some $H \in \mathcal{L}(X)$. It is clear that $H$ is a projection and commutes with all members of $\mathcal{M}$. Since $\mathcal{M}$ is $\sigma$-complete, the projections

$$
Q=\liminf H_{n}=\bigvee_{n=1}^{\infty} \bigwedge_{k=n}^{\infty} H_{k}, \quad R=\limsup H_{n}=\bigwedge_{n=1}^{\infty} \bigvee_{k=n}^{\infty} H_{k}
$$

both belong to $\mathcal{M}$. We claim that $Q \leq H \leq R$. Indeed, the sequence $\left\{\widetilde{H}_{n}\right\}_{n=1}^{\infty}$, defined by $\widetilde{H}_{n}=\bigwedge_{k=n}^{\infty} H_{k}$, increases to $Q$ in the order of $\mathcal{M}$ and so, by Lemma XVII.3.4 in [6], $\widetilde{H}_{n} \rightarrow Q$ in $\mathcal{L}_{\mathrm{S}}(X)$. Since $\widetilde{H}_{n} \leq H_{n}$ (i.e., $\widetilde{H}_{n} H_{n}=\widetilde{H}_{n}$ ) for each $n=1,2, \ldots$ and both $\widetilde{H}_{n} \rightarrow Q$ and $H_{n} \rightarrow H$ in $\mathcal{L}_{\mathrm{S}}(X)$, we deduce that $Q H=Q$, that is, $Q \leq H$. A similar argument yields $H \leq R$, which proves our claim. Therefore, it is sufficient to show that $Q=R$. Since $\mathcal{M}$ is atomic, it suffices to prove that $P Q=P R$ for all atoms $P$ in $\mathcal{M}$.

Given a fixed atom $P \in \mathcal{M}$, we have $P H_{n} \rightarrow P H$ in $\mathcal{L}_{\mathrm{s}}(X)$ as $n \rightarrow \infty$. Since $P H_{n} \in\{0, P\}$ for all $n$, it follows that there exists $N \in \mathbb{N}$ such that either $P H_{n}=0$ or $P H_{n}=P$ for all $n \geq N$. In the first case,

$$
\begin{aligned}
P Q & =P\left(\liminf H_{n}\right)=\liminf \left(P H_{n}\right)=0 \\
& =\lim \sup \left(P H_{n}\right)=P\left(\lim \sup H_{n}\right)=P R,
\end{aligned}
$$

and similarly, in the second case we find that $P Q=P=P R$. Hence, in either case $P Q=P R$.

Remark 2.3. Let $X$ be a Banach space and $\mathcal{M} \subseteq \mathcal{L}(X)$ be a Bade $\sigma$-complete B.a. of projections. Suppose that $\left\{P_{n}\right\}_{n=1}^{\infty}$ is any sequence in $\mathcal{M}$ such that $P_{n} \rightarrow P$ in $\mathcal{L}_{\mathrm{s}}(X)$ as $n \rightarrow \infty$. Then $P$ is a projection and it commutes with all elements of $\mathcal{M}$. It follows from the proof of Theorem 2.2 that always

$$
\liminf P_{n} \leq P \leq \limsup P_{n},
$$

where $\liminf P_{n}$ and $\limsup P_{n}$ exist in $\mathcal{M}$. The proof of Theorem 2.2 is based on the fact that if, in addition, $\mathcal{M}$ is atomic, then necessarily $\liminf P_{n}=\limsup P_{n}$ always holds. This is not true in general for nonatomic $\mathcal{M}$, even if $\mathcal{M}$ is Bade complete; see Remark 4.5. 
In the remainder of this section we exhibit examples of Bade $\sigma$-complete B.a.'s of projections which are atomic but not Bade atomic. In view of Lemma 2.1(ii) such B.a.'s cannot be Bade complete. We discuss these examples in some detail, since we will use such types of B.a.'s again in other sections of the paper.

First we recall some terminology and facts from the theory of Banach lattices. For details we refer the reader to any of the books on this subject (e.g. [9]). Let $(Y,\|\cdot\|)$ be a Dedekind complete Banach lattice with positive cone $Y^{+}$. Then

$$
\mathcal{B}_{Y}=\left\{P \in \mathcal{L}(Y): P^{2}=P \text { and } 0 \leq P y \leq y \text { for all } y \in Y^{+}\right\}
$$

is the B.a. of all band projections in $Y$. Note that $\|P\| \leq 1$ for any $P \in \mathcal{B}_{Y}$. Since $Y$ is assumed to be Dedekind complete, $\mathcal{B}_{Y}$ is an abstractly complete B.a. of projections and has the property that an upwards directed system $\left\{P_{\alpha}\right\} \subseteq \mathcal{B}_{Y}$ increases to $P \in \mathcal{B}_{Y}$ in the order of $\mathcal{B}_{Y}$ if, and only if, $P_{\alpha} y \uparrow_{\alpha} P y$ in the order of $Y$ for every $y \in Y^{+}$. Moreover, each $P \in \mathcal{B}_{Y}$ is disjointness preserving (i.e., $\left|P y_{1}\right| \wedge\left|P y_{2}\right|=0$ whenever $\left|y_{1}\right| \wedge\left|y_{2}\right|=0$ in $Y$ ) and satisfies $|P y|=P|y|$ for every $y \in Y$. If, in addition, $Y$ has order continuous norm, then it follows that $\mathcal{B}_{Y}$ is Bade complete.

Let $(\Omega, \Sigma)$ be a measurable space and let $X=\mathrm{ca}(\Sigma)$ denote the (real) Banach space of all $\sigma$-additive measures $\mu: \Sigma \rightarrow \mathbb{R}$ with norm $\|\mu\|=|\mu|(\Omega)$, where $|\mu|$ denotes the variation measure of $\mu$. If $\mu, \nu \in X$, then we write $\mu \leq \nu$ whenever $\mu(E) \leq \nu(E)$ for all $E \in \Sigma$. Equipped with this order, $X$ is a Dedekind complete Banach lattice with order continuous norm. In fact, $X$ is an abstract $L$-space, meaning that $\|\mu+\nu\|=\|\mu\|+\|\nu\|$ whenever $\mu, \nu \in X^{+}$. We point out that two elements $\mu, \nu \in X$ are disjoint (i.e., $|\mu| \wedge|\nu|=0)$ if, and only if, $\mu \perp \nu$, that is, $\mu$ and $\nu$ are mutually singular as measures $([9$, p. 11] $)$. Moreover, we note that the principal band generated by an element $\mu \in \mathrm{ca}(\Sigma)$ consists precisely of those $\nu \in \mathrm{ca}(\Sigma)$ which are absolutely continuous with respect to $\mu$. This principal band will be denoted by $\mathrm{AC}(\mu)$. The positive cone of $\mathrm{ca}(\Sigma)$ is denoted by $\mathrm{ca}^{+}(\Sigma)$.

Let $Y \subseteq \mathrm{ca}(\Sigma)$ be any band. Then $Y$ is itself a Dedekind complete Banach lattice with order continuous norm. In particular, the B.a. $\mathcal{B}_{Y}$ of all band projections in $Y$ is a Bade complete B.a. Given $\mu \in \mathrm{ca}(\Sigma)$ and $E \in \Sigma$, let $\mu_{E} \in \mathrm{ca}(\Sigma)$ be defined by $\mu_{E}(F)=\mu(E \cap F)$ for all $F \in \Sigma$. Since $\left|\mu_{E}\right|=|\mu|_{E} \leq|\mu|$ and $Y$ is solid in ca $(\Sigma)$, it follows that $\mu_{E} \in Y$ whenever $\mu \in Y$ and $E \in \Sigma$. Given $E \in \Sigma$, the mapping $P_{Y}(E): Y \rightarrow Y$ defined by

$$
P_{Y}(E) \mu=\mu_{E}, \quad \mu \in Y
$$

is a band projection in $Y$. The map $P_{Y}: \Sigma \rightarrow \mathcal{L}(Y)$ given by $E \mapsto P_{Y}(E)$ for $E \in \Sigma$ is clearly finitely additive, multiplicative (i.e., $P_{Y}(E \cap F)=$ $P_{Y}(E) P_{Y}(F)$ for all $\left.E, F \in \Sigma\right)$ and $P_{Y}(\Omega)=I$. Actually, $P_{Y}$ is $\sigma$-additive in $\mathcal{L}_{\mathrm{s}}(Y)$ because $\left\|P_{Y}(E) \mu\right\|=\left|\mu_{E}\right|(\Omega) \leq|\mu|(E)$ for $E \in \Sigma$, and each $\mu \in Y$ 
is $\sigma$-additive on $\Sigma$. Hence, $P_{Y}$ is a spectral measure in $Y$. It follows that the range $\mathcal{M}_{Y}=P_{Y}(\Sigma)$ of $P_{Y}$ is a Bade $\sigma$-complete B.a. in $\mathcal{L}(Y)$ (see Corollary XVII.3.10 in [6]). In particular, $\mathcal{M}_{Y}$ is a $\sigma$-subalgebra (in the sense of [16, Section 23]) of the Bade complete B.a. $\mathcal{B}_{Y}$ of all band projections in $Y$. For the case $Y=\operatorname{ca}(\Sigma)$ we will omit the subscript $Y$ and simply write $\mathcal{M}$ and $P$.

Lemma 2.4. Let $(\Omega, \Sigma)$ be a measurable space, $Y$ a band in $\mathrm{ca}(\Sigma)$ and $\mathcal{M}_{Y}=P_{Y}(\Sigma)$ be the range of the spectral measure $P_{Y}: \Sigma \rightarrow \mathcal{L}_{\mathrm{s}}(Y)$ given by (1). Then $\mathcal{B}_{Y}=\overline{\mathcal{M}}_{Y}$, the $\tau_{\mathrm{s}}$-closure of $\mathcal{M}_{Y}$ in $\mathcal{L}(Y)$.

Proof. The closedness of $\mathcal{B}_{Y}$ in $\mathcal{L}_{\mathrm{S}}(Y)$ can easily be verified directly in this situation; it also follows from the Bade completeness of $\mathcal{B}_{Y}$ by Corollary XVII.3.7 in [6]. Hence, $\overline{\mathcal{M}}_{Y} \subseteq \mathcal{B}_{Y}$.

An application of the Hahn decomposition theorem yields the following:

FACT 1. If $Q \in \mathcal{B}_{Y}$ and $\mu \in Y$, then there exists $A \in \Sigma$ with $P_{Y}(A) \mu$ $=Q \mu$.

As a consequence we also have:

FACT 2. If $Q \in \mathcal{B}_{Y}$ and $\mu_{1}, \ldots, \mu_{n} \in Y$, then there exists $A \in \Sigma$ such that

$$
P_{Y}(A) \mu_{j}=Q \mu_{j}, \quad j=1, \ldots, n .
$$

Indeed, define $\mu=\left|\mu_{1}\right| \vee \cdots \vee\left|\mu_{n}\right|$. By Fact 1 , there exists $A \in \Sigma$ with $P_{Y}(A) \mu=Q \mu$. Observe that, since both $P_{Y}(A)$ and $Q$ are band projections, the collection of all $\nu \in Y$ satisfying $P_{Y}(A) \nu=Q \nu$ is a band in $Y$. In particular, $P_{Y}(A) \mu=Q \mu$ implies that $P_{Y}(A) \nu=Q \nu$ for all $\nu \in Y$ satisfying $|\nu| \leq \mu$. This establishes Fact 2 .

To complete the proof of the lemma, fix $Q \in \mathcal{B}_{Y}$. For any finite subset $F \subseteq Y$, Fact 2 guarantees the existence of a set $A_{F} \in \Sigma$ such that $P_{Y}\left(A_{F}\right) \mu=Q \mu$ for all $\mu \in F$. Directing the collection $\mathcal{F}$ of all finite subsets of $Y$ by inclusion, it is now clear that the net $\left\{P_{Y}\left(A_{F}\right): F \in \mathcal{F}\right\}$ is $\tau_{\mathrm{s}}$-convergent to $Q$. Accordingly, $Q \in \overline{\mathcal{M}}_{Y}$ and so $\mathcal{B}_{Y} \subseteq \overline{\mathcal{M}}_{Y}$.

EXAmPle 2.5. A Bade $\sigma$-complete B.a. of projections which is atomic, but not Bade atomic.

Let $\Omega=[0,1]$ and $\Sigma$ be the $\sigma$-algebra of all Borel subsets of $\Omega$. Let $Y=\mathrm{ca}(\Sigma)$ and $\mathcal{M}=P(\Sigma)$ with $P=P_{Y}$ given by (1). The atoms in $\mathcal{M}$ are all projections of the form $P(\{w\})$ with $w \in \Omega$. If $\emptyset \neq E \in \Sigma$, then $P(\{w\}) \leq P(E)$ for all $w \in E$, so it is clear that $\mathcal{M}$ is an atomic B.a.

Let $\lambda \in \mathrm{ca}(\Sigma)$ denote Lebesgue measure (or any other non-zero, continuous measure). Since $P(\{w\}) \lambda=0$ for every $w \in \Omega$, it is clear that $I$ cannot be the $\tau_{\mathrm{s}}$-limit of sums of the form $\sum_{w \in F} P(\{w\})=P(F)$ with $F \subseteq \Omega$ a finite set. Hence, $\mathcal{M}$ is not Bade atomic. 
In view of Lemma 2.1(ii) we see that $\mathcal{M}$ cannot be Bade complete. This also follows immediately from Lemma 2.4 . Indeed, it is easily verified that the band projection $P_{\lambda} \in \mathcal{B}_{Y}=\overline{\mathcal{M}}$ onto the principal band $\mathrm{AC}(\lambda)$ generated by $\lambda$ does not belong to $\mathcal{M}$.

We conclude this section with some comments about the connection between $\mathcal{M}$ and its $\tau_{\mathrm{s}}$-closure $\overline{\mathcal{M}}$ being Bade atomic. The following observation is routine to verify.

Lemma 2.6. Let $X$ be a Banach space and $\mathcal{M} \subseteq \mathcal{L}(X)$ be a Bade $\sigma$ complete B.a. of projections. If $P \in \mathcal{M}$ is an atom, then $P$ is also an atom in the Bade complete B.a. $\overline{\mathcal{M}}$.

The next result is a consequence of Lemma 2.6 and of Lemma XVII.3.4 in [6], together with the fact that if $\left\{P_{\alpha}\right\}_{\alpha \in \mathcal{A}}$ is the collection of all the atoms in a Bade $\sigma$-complete B.a. $\mathcal{M}$, then $\mathcal{M}$ is Bade atomic if, and only if, $I=\sum_{\alpha \in \mathcal{A}} P_{\alpha}$ as a $\tau_{\mathrm{s}}$-summable series in $\mathcal{L}_{\mathrm{s}}(X)$. We leave the details to the reader.

Proposition 2.7. Let $X$ be a Banach space and $\mathcal{M} \subseteq \mathcal{L}(X)$ be a Bade $\sigma$-complete B.a. of projections. Then $\mathcal{M}$ is Bade atomic if, and only if, $\overline{\mathcal{M}}$ is atomic and every atom of $\overline{\mathcal{M}}$ belongs to $\mathcal{M}$.

We remark that both requirements on $\overline{\mathcal{M}}$ are necessary. Indeed, the B.a. $\mathcal{M}$ of Example 2.5 is not Bade atomic, all atoms of $\overline{\mathcal{M}}=\mathcal{B}_{Y}$ belong to $\mathcal{M}$ and $\overline{\mathcal{M}}$ is not atomic. On the other hand, let $\Omega=[0,1]$ and $\Sigma$ denote the $\sigma$-algebra of all subsets of $\Omega$ which are either countable or co-countable. Again let $Y=\mathrm{ca}(\Sigma)$ and $\mathcal{M}=P(\Sigma)$, where $P=P_{Y}$ is the spectral measure given by (1). Denote by $\lambda$ the measure on $\Sigma$ which takes the value 0 on all countable sets and the value 1 on all co-countable sets. It turns out that $\mathrm{ca}(\Sigma) \cong \ell^{1}(\Omega) \oplus\langle\lambda\rangle$, where $\langle\lambda\rangle$ denotes the 1-dimensional subspace generated by $\lambda$. Let $P_{\mathrm{c}}$ denote the (band) projection in $\operatorname{ca}(\Sigma)$ onto $\langle\lambda\rangle$. It can be verified that $\mathcal{M}$ is an atomic Bade $\sigma$-complete B.a. (the atoms in $\mathcal{M}$ are all projections of the form $P(\{w\})$ with $w \in \Omega$ ), but is not Bade atomic. In this case, $\overline{\mathcal{M}}$ is atomic as well (the atoms in $\overline{\mathcal{M}}$ are the atoms in $\mathcal{M}$ together with $P_{\mathrm{c}}$ ), but an additional atom $P_{\mathrm{c}}$ exists in $\overline{\mathcal{M}}$ which does not occur in $\mathcal{M}$.

3. Criteria for sequential closedness. Theorem 2.2 raises the question of whether or not there is a similar result for Bade $\sigma$-complete B.a.'s which are not atomic. In the final section this will be answered using the criteria of this section.

Let $(\Omega, \Sigma)$ be a measurable space and $\Phi \subseteq \mathrm{ca}^{+}(\Sigma)$. The $\sigma$-algebra $\Sigma$ is said to be sequentially $\Phi$-complete if, whenever $\left\{A_{n}\right\}_{n=1}^{\infty} \subseteq \Sigma$ satisfies $\nu\left(A_{n} \triangle A_{m}\right) \rightarrow 0$ as $n, m \rightarrow \infty$ for every $\nu \in \Phi$, then there exists $A \in \Sigma$ 
with $\nu\left(A \triangle A_{n}\right) \rightarrow 0$ as $n \rightarrow \infty$ for every $\nu \in \Phi$. Of course, $B \triangle C$ denotes the symmetric difference of any pair of subsets $B, C$ of $\Omega$.

Given any $\Phi \subseteq \mathrm{ca}^{+}(\Sigma)$, let $X_{\Phi}$ denote the band generated by $\Phi$ in ca $(\Sigma)$ and let $X_{\Phi}^{\prime}$ denote the dual space of $X_{\Phi}$, which is itself a Banach lattice. The absolute weak-star topology $|\sigma|\left(X_{\Phi}^{\prime}, X_{\Phi}\right)$ on $X_{\Phi}^{\prime}$ is that determined by the family of seminorms $\left\{q_{\nu}: \nu \in X_{\Phi}\right\}$, where

$$
q_{\nu}(\xi)=\langle|\nu|,|\xi|\rangle, \quad \xi \in X_{\Phi}^{\prime}
$$

Each bounded $\Sigma$-measurable function $g$ on $\Omega$ defines an element of $X_{\Phi}^{\prime}$ by

$$
\langle\nu, g\rangle=\int_{\Omega} g d \nu, \quad \nu \in X_{\Phi}
$$

and

$$
q_{\nu}(g)=\langle|\nu|,|g|\rangle=\int_{\Omega}|g| d|\nu|
$$

for each $\nu \in X_{\Phi}$. For $Y=X_{\Phi}$, the spectral measure defined by (1) is denoted simply by $P_{\Phi}: \Sigma \rightarrow \mathcal{L}\left(X_{\Phi}\right)$, in which case $\mathcal{M}_{\Phi}=P_{\Phi}(\Sigma)$ is a Bade $\sigma$-complete B.a. of projections in $X_{\Phi}$.

We require the following technical result. Recall if $P, Q$ are two commuting projections in a Banach space $X$, then $P \triangle Q$ is defined to be the projection $P(I-Q)+(I-P) Q$ which, in turn, equals $(P \vee Q)-(P \wedge Q)$ whenever $P, Q$ are elements of a B.a. of projections on $X$ (since $P \vee Q=P+Q-P Q$ and $P \wedge Q=P Q)$.

Lemma 3.1. Let $X$ be a Banach space and $\mathcal{M} \subseteq \mathcal{L}(X)$ be a B.a. such that $M=\sup \{\|P\|: P \in \mathcal{M}\}<\infty$. Let $P, Q \in \mathcal{M}$. Then, for each $x \in X$, we have

$$
(2 M)^{-1}\|P x-Q x\| \leq\|(P \triangle Q) x\| \leq 2 M\|P x-Q x\| .
$$

Proof. Suppose first that $P Q=0$. Then

$$
\|(P+Q) x\|=\|(P-Q)(P x-Q x)\| \leq 2 M\|P x-Q x\| .
$$

Also, since $P-Q=(P-Q)(P+Q)$, it follows that

$$
\|P x-Q x\| \leq\|P-Q\|\|P x+Q x\| \leq 2 M\|P x+Q x\| .
$$

Now suppose that $P, Q$ are arbitrary. Since $(P-P Q)(Q-P Q)=0$ we see from the above that

$$
\begin{aligned}
\|P x-Q x\| & =\|(P-P Q) x-(Q-P Q) x\| \\
& \leq 2 M\|(P-P Q) x+(Q-P Q) x\|=2 M\|(P \triangle Q) x\| .
\end{aligned}
$$

Similarly,

$$
\begin{aligned}
\|(P \triangle Q) x\| & =\|(P-P Q) x+(Q-P Q) x\| \\
& \leq 2 M\|(P-P Q) x-(Q-P Q) x\|=2 M\|P x-Q x\| .
\end{aligned}
$$


In the following theorem some characterizations of sequential $\Phi$-completeness of $\Sigma$ are presented.

Theorem 3.2. Let $(\Omega, \Sigma)$ be a measurable space and $\Phi \subseteq \mathrm{ca}^{+}(\Sigma)$. The following statements are equivalent.

(i) $\Sigma$ is sequentially $\Phi$-complete.

(ii) $\left\{\chi_{A}: A \in \Sigma\right\} \subseteq X_{\Phi}^{\prime}$ is sequentially complete for the $|\sigma|\left(X_{\Phi}^{\prime}, X_{\Phi}\right)$ topology.

(iii) The Bade $\sigma$-complete B.a. $\mathcal{M}_{\Phi}$ is sequentially closed in $\mathcal{L}_{\mathrm{s}}\left(X_{\Phi}\right)$.

Proof. (i) $\Rightarrow$ (iii). Let $\left\{P_{\Phi}\left(A_{n}\right)\right\}_{n=1}^{\infty} \subseteq \mathcal{M}_{\Phi}$ converge to $P$ in $\mathcal{L}_{\mathrm{s}}\left(X_{\Phi}\right)$, in which case $\left\{P_{\Phi}\left(A_{n}\right)\right\}_{n=1}^{\infty}$ is Cauchy in $\mathcal{L}_{\mathrm{s}}\left(X_{\Phi}\right)$. Lemma 3.1 implies that $\left\|P_{\Phi}\left(A_{n} \triangle A_{m}\right) \mu\right\| \rightarrow 0$ as $m, n \rightarrow \infty$ for every $\mu \in X_{\Phi}$. In particular, for all $\nu \in \Phi \subseteq X_{\Phi}$, we see that

$$
0 \leq \nu\left(A_{n} \triangle A_{m}\right)=\left\|\nu_{A_{n} \triangle A_{m}}\right\|=\left\|P_{\Phi}\left(A_{n} \triangle A_{m}\right) \nu\right\| \rightarrow 0
$$

as $m, n \rightarrow \infty$. By hypothesis there is $A \in \Sigma$ such that

$$
\left\|P_{\Phi}\left(A_{n} \triangle A\right) \nu\right\|=\nu\left(A_{n} \triangle A\right) \rightarrow 0, \quad \nu \in \Phi,
$$

as $n \rightarrow \infty$. The same conclusion holds for any $\mu \in \operatorname{ca}(\Sigma)$ satisfying $|\mu| \leq$ $\sum_{j=1}^{\infty} c_{j} \nu_{j}$ for some $0 \leq c_{j} \in \mathbb{R}$ and $\nu_{j} \in \Phi$ and, by a standard approximation argument and using Lemma 3.1, it follows that $P_{\Phi}\left(A_{n}\right) \rightarrow P_{\Phi}(A)$ in $\mathcal{L}_{\mathrm{S}}\left(X_{\Phi}\right)$. Accordingly, $P=P_{\Phi}(A) \in \mathcal{M}_{\Phi}$.

(iii) $\Rightarrow$ (ii). Let $\left\{\chi_{A_{n}}\right\}_{n=1}^{\infty} \subseteq X_{\Phi}^{\prime}$ be $|\sigma|\left(X_{\Phi}^{\prime}, X_{\Phi}\right)$-Cauchy. The identities $q_{\mu}\left(\chi_{A_{n}}-\chi_{A_{m}}\right)=|\mu|\left(A_{n} \triangle A_{m}\right)$, for each $\mu \in X_{\Phi}$, together with Lemma 3.1 , imply that $\left\{P_{\Phi}\left(A_{n}\right)\right\}_{n=1}^{\infty}$ is Cauchy in $\mathcal{L}_{\mathrm{S}}\left(X_{\Phi}\right)$. Hence, by sequential completeness of $\mathcal{L}_{\mathrm{S}}\left(X_{\Phi}\right)$ and sequential closedness of $\mathcal{M}_{\Phi}$, there is $A \in \Sigma$ such that $P_{\Phi}\left(A_{n}\right) \rightarrow P_{\Phi}(A)$ in $\mathcal{L}_{\mathrm{S}}\left(X_{\Phi}\right)$. Arguing as above, it follows that $\chi_{A_{n}} \rightarrow \chi_{A}$ in $X_{\Phi}^{\prime}$ with respect to $|\sigma|\left(X_{\Phi}^{\prime}, X_{\Phi}\right)$.

(ii) $\Rightarrow$ (i). Let $\left\{A_{n}\right\}_{n=1}^{\infty} \subseteq \Sigma$ satisfy $\nu\left(A_{n} \triangle A_{m}\right) \rightarrow 0$ as $m, n \rightarrow \infty$ for all $\nu \in \Phi$. Via a routine argument (similar to the one used above), it follows that actually $|\mu|\left(A_{n} \triangle A_{m}\right) \rightarrow 0$ as $m, n \rightarrow \infty$ for every $\mu \in X_{\Phi}$. Hence, $\left\{\chi_{A_{n}}\right\}_{n=1}^{\infty}$ is a $|\sigma|\left(X_{\Phi}^{\prime}, X_{\Phi}\right)$-Cauchy sequence in $X_{\Phi}^{\prime}$ and so, by hypothesis, there exists $A \in \Sigma$ such that $\chi_{A_{n}} \rightarrow \chi_{A}$ with respect to $|\sigma|\left(X_{\Phi}^{\prime}, X_{\Phi}\right)$. In particular, for each $\nu \in \Phi$ we conclude that

$$
\nu\left(A \triangle A_{n}\right)=q_{\nu}\left(\chi_{A}-\chi_{A_{n}}\right) \rightarrow 0, \quad n \rightarrow \infty,
$$

that is, $\Sigma$ is sequentially $\Phi$-complete.

Let $\mathcal{M} \subseteq \mathcal{L}(X)$ be a Bade $\sigma$-complete B.a. of projections in a Banach space $X$. Any spectral measure $P: \Sigma \rightarrow \mathcal{L}_{\mathrm{s}}(X)$, defined on some measurable space $(\Omega, \Sigma)$ and satisfying $P(\Sigma)=\mathcal{M}$, is said to represent $\mathcal{M}$. Such spectral measures always exist (see e.g. [6, Corollary XVII.3.10]), but are surely not unique. Given $x \in X$, any $x^{\prime} \in X^{\prime}$ with the properties: 
(i) $\left\langle B x, x^{\prime}\right\rangle \geq 0$ for all $B \in \mathcal{M}$,

(ii) $\left\langle B x, x^{\prime}\right\rangle=0$ for some $B \in \mathcal{M}$ if and only if $B x=0$,

is called a Bade functional for $x$ (relative to $\mathcal{M}$ ); see [6, Lemma XVII.3.12]. In this case, the set function $\left\langle P(\cdot) x, x^{\prime}\right\rangle: E \mapsto\left\langle P(E) x, x^{\prime}\right\rangle$ for $E \in \Sigma$ belongs to $\mathrm{ca}^{+}(\Sigma)$ and is mutually absolutely continuous to the $X$-valued vector measure $P(\cdot) x: E \mapsto P(E) x$ for $E \in \Sigma$. In view of property (ii), for each $x \in X$ the measures $\left\{\left\langle P(\cdot) x, x^{\prime}\right\rangle: x^{\prime} \in B(P ; x)\right\}$, where

$$
B(P ; x)=\left\{x^{\prime} \in X^{\prime}: x^{\prime} \text { is a Bade functional for } x\right\}
$$

are all mutually absolutely continuous. Any family of measures of the form

$$
B(P)=\left\{\left\langle P(\cdot) x, \varphi_{x}\right\rangle: x \in X, \varphi_{x} \in B(P ; x)\right\} \subseteq \mathrm{ca}^{+}(\Sigma)
$$

is called a Bade family relative to $P$.

TheOrem 3.3. Let $X$ be a Banach space and $\mathcal{M} \subseteq \mathcal{L}(X)$ be a Bade $\sigma$ complete B.a. of projections. Then $\mathcal{M}$ is sequentially closed in $\mathcal{L}_{\mathrm{s}}(X)$ if , and only if, for every (for some) representing spectral measure $P: \Sigma \rightarrow \mathcal{L}(X)$ for $\mathcal{M}$ and for every (for some) Bade family of measures $B(P)$ relative to $P$, the $\sigma$-algebra $\Sigma$ is sequentially $B(P)$-complete.

Proof. Suppose that $P$ and $B(P)$ exist with the stated property. Let $P_{n}=P\left(A_{n}\right)$ for $n=1,2, \ldots$ be a sequence in $\mathcal{M}=P(\Sigma)$ which is $\tau_{\mathrm{s}^{-}}$ convergent to $Q \in \mathcal{L}(X)$. Fix $x \in X$ and, for $\varphi_{x} \in B(P ; x)$ as in (2), let $\nu_{x}=\left\langle P(\cdot) x, \varphi_{x}\right\rangle$. Since $\left\{P_{n} x\right\}_{n=1}^{\infty}$ is Cauchy in $X$, it follows from Lemma 3.1 that $\left\|\left(P_{n} \triangle P_{m}\right) x\right\| \rightarrow 0$ as $m, n \rightarrow \infty$. Hence, $\nu_{x}\left(A_{n} \triangle A_{m}\right) \rightarrow 0$ as $m, n \rightarrow \infty$. This holds for every $x \in X$ and so, by hypothesis, there exists $A \in \Sigma$ such that $\nu_{x}\left(A_{n} \triangle A\right) \rightarrow 0$ as $n \rightarrow \infty$ for every $x \in X$. Since the vector measure $P(\cdot) x$ is absolutely continuous with respect to $\nu_{x}$, it follows from a theorem of Pettis (see [5, p. 10]), that also $\left\|P\left(A_{n} \triangle A\right) x\right\| \rightarrow 0$ as $n \rightarrow \infty$. In view of Lemma 3.1 we conclude that $P\left(A_{n}\right) x \rightarrow P(A) x$ in $X$ as $n \rightarrow \infty$ for every $x \in X$. Hence, $P\left(A_{n}\right) \rightarrow P(A)$ in $\mathcal{L}_{\mathrm{s}}(X)$ and so $Q=P(A) \in \mathcal{M}$. This shows that $\mathcal{M}$ is sequentially closed in $\mathcal{L}_{\mathrm{s}}(X)$.

Conversely, suppose that $\mathcal{M}$ is sequentially closed in $\mathcal{L}_{\mathrm{S}}(X)$. Let $P$ : $\Sigma \rightarrow \mathcal{L}(X)$ be any representing spectral measure for $\mathcal{M}$ and $B(P)$ be any Bade family of measures relative to $P$. We have to show that $\Sigma$ is sequentially $B(P)$-complete. So, let $\left\{A_{n}\right\}_{n=1}^{\infty} \subseteq \Sigma$ satisfy $\nu_{x}\left(A_{n} \triangle A_{m}\right) \rightarrow 0$ as $n, m \rightarrow \infty$ for all $x \in X$ (with notation $\nu_{x}$ as above). Using the Pettis theorem mentioned above and Lemma 3.1 we can conclude that $\left\{P\left(A_{n}\right)\right\}_{n=1}^{\infty}$ $\subseteq \mathcal{M}$ is Cauchy in $\mathcal{L}_{\mathrm{s}}(X)$. Since $\mathcal{L}_{\mathrm{S}}(X)$ is sequentially complete and $\mathcal{M}$ is $\tau_{\mathrm{s}}$-closed, there exists $A \in \Sigma$ such that $P\left(A_{n}\right) \rightarrow P(A)$ in $\mathcal{L}_{\mathrm{s}}(X)$. It follows easily that $\nu_{x}\left(A_{n} \triangle A\right) \rightarrow 0$ as $n \rightarrow \infty$ for every $x \in X$.

4. Examples. In this section we apply the results of the previous section to various examples. The idea is to find suitable measurable spaces $(\Omega, \Sigma)$ 
together with families of measures $\Phi \subseteq \mathrm{ca}^{+}(\Sigma)$ such that $\Sigma$ is (or is not) sequentially $\Phi$-complete.

4.1. ExAmple A. There exists a non-atomic, Bade $\sigma$-complete B.a. of projections which is sequentially $\tau_{\mathrm{s}}$-closed, but not $\tau_{\mathrm{s}}$-closed.

Let $\Omega=[0,1]$ and $\Sigma$ be the $\sigma$-algebra of all Borel subsets of $\Omega$. We now let $\Phi \subseteq \mathrm{ca}^{+}(\Sigma)$ be the family of all non-negative, continuous measures on $\Sigma$. Then $X_{\Phi}=\Phi-\Phi$ is the band in ca $(\Sigma)$ consisting of all continuous measures on $\Sigma$ (i.e., a measure $\mu \in \operatorname{ca}(\Sigma)$ belongs to $X_{\Phi}$ if, and only if, $\mu(\{w\})=0$ for all $w \in \Omega$ ). Before proceeding, it will be convenient to recall the following fact, which indicates that $X_{\Phi}$ contains an abundance of measures.

LEMMA 4.1. Let $F$ be an uncountable Borel subset of $\Omega=[0,1]$. Then there exists $0<\mu \in \Phi$ satisfying $\mu(\Omega \backslash F)=0$.

Proof. Two sets $B_{1}, B_{2} \in \Sigma$ are called Borel isomorphic if there is a bijection $\varphi: B_{1} \rightarrow B_{2}$ such that both $\varphi$ and $\varphi^{-1}$ are Borel measurable (see e.g. [12, p. 7]). In particular, if $\nu$ is a continuous Borel measure on $B_{1}$, then the image measure $\widetilde{\nu}: E \mapsto \nu\left(\varphi^{-1}(E)\right)$, for each Borel set $E \subseteq B_{2}$, is clearly continuous on $B_{2}$.

Now choose $B_{1}=\Omega$ and $B_{2}=F$, in which case there exists a Borel isomorphism $\varphi: \Omega \rightarrow F$ (see Theorems 2.8 and 2.12 in [12], for example). Let $\lambda$ be Lebesgue measure on $\Omega$ and define the image measure $\tilde{\lambda}$ on $F$ as above. Then the measure $\mu: E \mapsto \widetilde{\lambda}(E \cap F)$, for $E \in \Sigma$, has the required properties.

For $Y=X_{\Phi}$, let $P_{\Phi}=P_{X_{\Phi}}: \Sigma \rightarrow \mathcal{L}\left(X_{\Phi}\right)$ be the spectral measure given by (1) and define $\mathcal{M}_{\Phi}=P_{\Phi}(\Sigma)$, which is a Bade $\sigma$-complete B.a. in $\mathcal{L}\left(X_{\Phi}\right)$. It follows from Lemma 4.1 that the $P_{\Phi}$-null sets are precisely the countable subsets of $\Omega$. Moreover, it is easy to see that $\mathcal{M}_{\Phi}$ is non-atomic. From Lemma 2.4 we know that $\overline{\mathcal{M}}_{\Phi}=\mathcal{B}_{X_{\Phi}}$, the B.a. of all band projections in $X_{\Phi}$. For any $\mu \in X_{\Phi}$ we denote by $Q_{\mu} \in \mathcal{B}_{X_{\Phi}}$ the band projection onto the principal band $\mathrm{AC}(\mu)$ generated by $\mu$. We will show that $Q_{\mu} \notin \mathcal{M}_{\Phi}$, for which we need the following observation.

LEMMA 4.2. Suppose that $\mu \in \Phi$ satisfies $\mu(E)>0$ for some set $E \in \Sigma$. Then there exists an uncountable Borel set $F \subseteq E$ such that $\mu(F)=0$.

Proof. We consider the case that $\mu=\lambda$ is Lebesgue measure on $\Omega=$ $[0,1]$. The general case then follows from [15] (Theorem 9 in Section 3 of Chapter 15). Now $\lambda(E)>0$ and so we can choose a compact set $C \subseteq E$ with $\lambda(C)>0$. Since $C$ is clearly an uncountable $G_{\delta}$-set, it follows from [10, Lemma 5.1] that there exists a nowhere dense, closed set $F \subseteq C$ with $\lambda(F)=0$ such that $F$ can be mapped continuously onto $\Omega$. In particular, $F$ must be uncountable. 
Lemma 4.3. For any $0<\mu \in \Phi$ we have $Q_{\mu} \notin \mathcal{M}_{\Phi}$. In particular, $\mathcal{M}_{\Phi}$ is not $\tau_{\mathrm{s}}$-closed in $\mathcal{L}(X)$, and hence, is also not Bade complete.

Proof. Fix $0<\mu \in \Phi$ and suppose that $Q_{\mu} \in \mathcal{M}_{\Phi}$, i.e., $Q_{\mu}=P_{\Phi}(E)$ for some $E \in \Sigma$. Since $\mu(E)=\mu(\Omega)>0$, it follows from Lemma 4.2 that there exists an uncountable Borel set $F \subseteq E$ such that $\mu(F)=0$. By Lemma 4.1 there is $0<\nu \in \Phi$ such that $\nu(\Omega \backslash F)=0$. Since $P_{\Phi}(F) \nu=\nu$ and $P_{\Phi}(F) \mu=\mu$, it is clear that $\nu \perp \mu$ and so $Q_{\mu} \nu=0$. However, this contradicts the fact that also $Q_{\mu} \nu=P_{\Phi}(E) \nu=P_{\Phi}(E) P_{\Phi}(F) \nu=P_{\Phi}(F) \nu=\nu$.

To finish the example, it remains to establish the following:

Proposition 4.4. The B.a. $\mathcal{M}_{\Phi}$ is sequentially closed in $\mathcal{L}_{\mathrm{S}}\left(X_{\Phi}\right)$.

Proof. By Theorem 3.2 it suffices to show that $\Sigma$ is sequentially $\Phi$ complete. This will be achieved in a number of steps. So, let $\{A(n)\}_{n=1}^{\infty} \subseteq \Sigma$ be any sequence such that $\mu(A(n) \triangle A(m)) \rightarrow 0$ as $m, n \rightarrow \infty$ for all $\mu \in \Phi$. Accordingly, the sequence (of equivalence classes of) $\left\{\chi_{A(n)}\right\}_{n=1}^{\infty}$ is Cauchy in $L^{1}(\mu)$, and so converges in $L^{1}(\mu)$ for every $\mu \in \Phi$.

STEP 1. For every $\nu \in \mathrm{ca}^{+}(\Sigma)$, the sequence $\left\{\chi_{A(n)}\right\}_{n=1}^{\infty}$ has a $\nu$-a.e. convergent subsequence.

To see this, let $\left\{w_{k}\right\}_{k=1}^{\infty}=\{w \in[0,1]: \nu(\{w\})>0\}$ be the (at most countable) set of atoms of $\nu$. By the usual diagonalization process we can find a subsequence $\left\{\chi_{A\left(n_{j}\right)}\right\}_{j=1}^{\infty}$ which converges at each of the points $w_{k}$ $(k=1,2, \ldots)$. Now decompose $\nu=\nu_{\mathrm{c}}+\nu_{\mathrm{a}}$ with $\nu_{\mathrm{c}} \in \Phi$ and $\nu_{\text {a }}$ purely atomic. Since the sequence $\left\{\chi_{A\left(n_{j}\right)}\right\}_{j=1}^{\infty}$ is convergent in $L^{1}\left(\nu_{\mathrm{c}}\right)$, it has a further subsequence converging $\nu_{\mathrm{c}}$-a.e. This final subsequence has the property that it converges $\nu$-a.e. This establishes Step 1.

For any $\nu \in \mathrm{ca}^{+}(\Sigma)$, let $M_{\nu}([0,1])$ denote the locally convex Hausdorff space of all $\mathbb{R}$-valued, $\nu$-measurable functions defined on $[0,1]$, equipped with the topology of pointwise convergence on $[0,1]$.

STEP 2. Given $\nu \in \mathrm{ca}^{+}(\Sigma)$, the sequence $\left\{\chi_{A(n)}\right\}_{n=1}^{\infty}$ has a cluster point in $M_{\nu}([0,1])$.

By Step 1 there is a subsequence $\left\{\chi_{A\left(n_{k}\right)}\right\}_{k=1}^{\infty}$, a Borel function $f$ and a set $K \in \Sigma$ with $\nu\left(K^{\mathrm{c}}\right)=0$ such that

$$
\chi_{A\left(n_{k}\right)} \rightarrow f \quad \text { pointwise on } K \text { as } k \rightarrow \infty \text {. }
$$

Since $\left\{\chi_{A\left(n_{k}\right)}\right\}_{k=1}^{\infty}$ is contained in the compact set $[0,1]^{[0,1]}$, this sequence has a cluster point $g \in[0,1]^{[0,1]}$. By (3) it follows that $f \chi_{K}=g \chi_{K}$. This implies that $g \in M_{\nu}([0,1])$, and hence, $g$ is a cluster point of $\left\{\chi_{A(n)}\right\}_{n=1}^{\infty}$ in $M_{\nu}([0,1])$.

Since the result of Step 2 applies to every subsequence of $\left\{\chi_{A(n)}\right\}_{n=1}^{\infty}$, this shows that $\left\{\chi_{A(n)}\right\}_{n=1}^{\infty}$ is relatively countably compact in $M_{\nu}([0,1])$ for all 
$\nu \in \mathrm{ca}^{+}(\Sigma)$. It follows from $[3$, Theorem $4 \mathrm{D}]$ that $\left\{\chi_{A(n)}\right\}_{n=1}^{\infty}$ is relatively sequentially compact in $\mathbb{R}^{[0,1]}$, and hence, has a subsequence $\left\{\chi_{A\left(n_{j}\right)}\right\}_{j=1}^{\infty}$ which converges everywhere on $[0,1]$. Of course, the limit function is of the form $\chi_{A}$ for some $A \in \Sigma$ and, by the dominated convergence theorem, it is clear that $\mu\left(A\left(n_{j}\right) \triangle A\right) \rightarrow 0$ as $j \rightarrow \infty$ for all $\mu \in \Phi$. Since also $\mu(A(n) \triangle A(m)) \rightarrow 0$ as $m, n \rightarrow \infty$, we may conclude that $\mu(A(n) \triangle A) \rightarrow 0$ as $n \rightarrow \infty$, for every $\mu \in \Phi$. This completes the proof of the proposition.

Before leaving Example A, we return to Remark 2.3.

Remark 4.5. Let $(\Omega, \Sigma), \Phi, P_{\Phi}$ and $\mathcal{M}_{\Phi}=P_{\Phi}(\Sigma)$ still be as in Example A. Let $A_{0}=[0,1 / 2]$. Then define $A_{1}=[0,1 / 4)$ and $A_{2}=[1 / 4,1 / 2]$. Now form $A_{3}=[0,1 / 8), A_{4}=[1 / 8,1 / 4), A_{5}=[1 / 4,3 / 8)$ and $A_{6}=$ $[3 / 8,1 / 2]$, and continue in the obvious way. For each $n \geq 0$, define the set

$$
B_{n}=[1 / 2,1] \backslash\left\{x+1 / 2: x \in A_{n}\right\}
$$

and then the projection $P_{n}=P_{\Phi}\left(A_{n} \cup B_{n}\right) \in \mathcal{M}_{\Phi}$. It can be shown that $P_{n} \rightarrow P=P_{\Phi}([1 / 2,1])$ in $\mathcal{L}_{\mathrm{s}}\left(X_{\Phi}\right)$ as $n \rightarrow \infty$. Since $\liminf P_{n}=0$ and $\limsup P_{n}=I$ (formed in the order of $\mathcal{M}_{\Phi}$ or in $\overline{\mathcal{M}}_{\Phi}$ ), we see that

$$
\liminf P_{n}<P<\lim \sup P_{n} \text {. }
$$

4.2. Example B. There exists a Bade $\sigma$-complete B.a. of projections which fails to be sequentially $\tau_{\mathrm{s}}$-closed.

To produce Bade $\sigma$-complete B.a.'s of projections which fail to be sequentially $\tau_{\mathrm{s}}$-closed, we begin by exhibiting a measurable space $(\Omega, \Sigma)$ and a family of measures $\Phi \subseteq \mathrm{ca}^{+}(\Sigma)$ such that $\Sigma$ is not sequentially $\Phi$-complete.

For the rest of this section let $\Omega=\{0,1\}^{\mathbb{N}}$, equipped with the product topology, and $\Sigma=\mathcal{B}$ be the $\sigma$-algebra of all Borel subsets of $\Omega$. Elements $\xi \in \Omega$ will be denoted as $\xi=\left(\xi_{n}\right)_{n=0}^{\infty}$ with $\xi_{n} \in\{0,1\}$ for all $n \in \mathbb{N}=$ $\{0,1,2, \ldots\}$. For each $n \in \mathbb{N}$, let $D_{n}=\left\{\xi \in \Omega: \xi_{n}=1\right\}$ and define

$$
\Phi=\left\{\mu \in \mathrm{ca}^{+}(\mathcal{B}): \mu\left(D_{n} \triangle D_{m}\right) \rightarrow 0 \text { as } m, n \rightarrow \infty\right\} .
$$

Proposition 4.6. The Borel $\sigma$-algebra $\mathcal{B}$ is not sequentially $\Phi$-complete.

To establish Proposition 4.6 it suffices to show that there is no set $E \in \mathcal{B}$ with the property that $\mu\left(E \triangle D_{n}\right) \rightarrow 0$ as $n \rightarrow \infty$ for all $\mu \in \Phi$ or, equivalently, that there is no set $E \in \mathcal{B}$ satisfying $\mu\left(D_{n}\right) \rightarrow \mu(E)$ as $n \rightarrow \infty$ for all $\mu \in \Phi$. Define

$$
\begin{aligned}
& \mathcal{A}=\left\{\mu \in \mathrm{ca}^{+}(\mathcal{B}): \mu\left(D_{n}\right) \rightarrow 0 \text { as } n \rightarrow \infty\right\}, \\
& \mathcal{J}=\{A \in \mathcal{B}: \mu(A)=0 \text { for all } \mu \in \mathcal{A}\},
\end{aligned}
$$

in which case $\mathcal{A} \subseteq \Phi$ and $\mathcal{J}$ is a $\sigma$-ideal in the Boolean $\sigma$-algebra $\mathcal{B}$. Suppose that there does exist a set $E \in \mathcal{B}$ such that $\lim _{n \rightarrow \infty} \mu\left(D_{n}\right)=\mu(E)$ for all 
$\mu \in \Phi$. Then clearly $E \in \mathcal{J}$. Define a homeomorphic involution $\pi: \Omega \rightarrow \Omega$ by $\pi(\xi)=\left(1-\xi_{0}, 1-\xi_{1}, \ldots\right)$ for each $\xi \in \Omega$, and let $E^{\sharp}=\pi^{-1}(\Omega \backslash E)$. Using the observations that $\pi^{-1}\left(D_{n}\right)=D_{n}^{\mathrm{c}}$ for all $n \in \mathbb{N}$ and that

$$
\mu \pi^{-1}\left(D_{n} \triangle D_{m}\right)=\mu\left(D_{n} \triangle D_{m}\right) \rightarrow 0 \quad \text { as } m, n \rightarrow \infty
$$

for all $\mu \in \Phi$, where $\mu \pi^{-1}: A \mapsto \mu\left(\pi^{-1}(A)\right)$ for $A \in \mathcal{B}$ is the image measure, it follows that $\mu \pi^{-1} \in \Phi$ whenever $\mu \in \Phi$. Hence, $\mu \pi^{-1}\left(D_{n}\right) \rightarrow \mu \pi^{-1}(E)$ for all $\mu \in \Phi$, which implies in particular that $\mu \pi^{-1}(E)=\mu(\Omega)$ for all $\mu \in \mathcal{A}$ and so $E^{\sharp} \in \mathcal{J}$. Since $\Omega=E \cup \pi\left(E^{\sharp}\right)$ and $\Omega$ is a complete metric space, at least one of $E$ or $\pi\left(E^{\sharp}\right)$ is a non-meager set. But $\pi$ is a homeomorphism and so we can conclude that $E$ or $E^{\sharp}$ is non-meager and both $E, E^{\sharp} \in \mathcal{J}$. So, if such a set $E$ exists, then necessarily $\mathcal{J}$ contains at least one non-meager set. Hence, Proposition 4.6 follows from the following fact.

Proposition 4.7. Every element of $\mathcal{J}$ is a meager subset of $\Omega$.

The proof of this proposition will be via some lemmas. First we require some further notation.

The collections of all subsets and of all finite subsets of $\mathbb{N}$ are denoted by $\mathcal{P}(\mathbb{N})$ and $\mathcal{P}_{\mathrm{f}}(\mathbb{N})$, respectively. For $0 \leq m \leq n \in \mathbb{N}$, define $[m, n)=\{k \in$ $\mathbb{N}: m \leq k<n\}$. If $\xi \in \Omega$, define $\operatorname{supp}(\xi)=\left\{n \in \mathbb{N}: \xi_{n}=1\right\}$. The mapping $\xi \mapsto \operatorname{supp}(\xi)$ is a bijection between $\Omega$ and $\mathcal{P}(\mathbb{N})$. For $n \in \mathbb{N} \backslash\{0\}$ we will identify $\{0,1\}^{n}$ with a subset of $\Omega$ via the imbedding

$$
\left(\xi_{0}, \ldots, \xi_{n-1}\right) \mapsto\left(\xi_{0}, \ldots, \xi_{n-1}, 0,0, \ldots\right) .
$$

In particular, if $1 \leq m \leq n \in \mathbb{N}$, we consider $\{0,1\}^{m}$ as a subset of $\{0,1\}^{n}$. If $M \in \mathcal{P}(\mathbb{N})$ is non-empty, then $\xi_{M}$ denotes the restriction of $\xi$ to $M$. For $n \in \mathbb{N}$, the characteristic function $\chi_{[0, n)}$ is denoted simply by $\chi_{n}$.

By $\lambda$ we denote the canonical Haar measure on $\Omega$, i.e., the product measure

$$
\lambda=\bigotimes_{n=0}^{\infty} \lambda_{n},
$$

where $\lambda_{n}$ is the probability measure on $\{0,1\}$ satisfying $\lambda_{n}(\{0\})=\lambda_{n}(\{1\})=$ $1 / 2$ for all $n \in \mathbb{N}$.

Given $1 \leq n \in \mathbb{N}$ and $\delta \in\{0,1\}^{n}$ we define $G(n, \delta)=\left\{\xi \in \Omega: \chi_{n} \xi=\delta\right\}$. The collection

$$
\left\{G(n, \delta): n \in \mathbb{N} \backslash\{0\}, \delta \in\{0,1\}^{n}\right\}
$$

is a base for the topology in $\Omega$ consisting of sets which are simultaneously open and closed. For each $\xi \in \Omega$, the collection $\left\{G\left(n, \chi_{n} \xi\right)\right\}_{n=1}^{\infty}$ is a neighborhood base at $\xi$. If $n, m \in \mathbb{N} \backslash\{0\}, \delta \in\{0,1\}^{n}$ and $\varepsilon \in\{0,1\}^{m}$, then $G(m, \varepsilon) \subseteq G(n, \delta)$ if and only if $n \leq m$ and $\chi_{n} \varepsilon=\delta$. Moreover, if $G(m, \varepsilon) \cap G(n, \delta) \neq \emptyset$, then either $G(m, \varepsilon) \subseteq G(n, \delta)$ or $G(n, \delta) \subseteq G(m, \varepsilon)$. 
LEMMA 4.8. Let $M \subseteq \Omega$ be closed and nowhere dense and let $n \in \mathbb{N} \backslash\{0\}$ and $\delta \in\{0,1\}^{n}$ be given. For every integer $l \geq n$, there exists an integer $m \geq l$ and $\varepsilon \in\{0,1\}^{m}$ such that $\chi_{l} \varepsilon=\delta$ and $G(m, \varepsilon) \cap M=\emptyset$.

Proof. Since the interior $\operatorname{int}(M)$ is empty, there exists $\xi \in G(l, \delta) \backslash M$, and since $M$ is closed, it follows that there exists $m \in \mathbb{N} \backslash\{0\}$ such that $G\left(m, \chi_{m} \xi\right) \subseteq G(l, \delta) \backslash M$. As observed above, this implies that $l \leq m$ and $\chi_{l} \varepsilon=\delta$, so we can take $\varepsilon=\chi_{m} \xi$.

LEMMA 4.9. Let $M \subseteq \Omega$ be closed and nowhere dense and let $n \in \mathbb{N} \backslash\{0\}$ and $\delta_{j} \in\{0,1\}^{n}$ for $j=0,1, \ldots, k$ be given. Then there exists an integer $m \geq n$ and elements $\varepsilon_{j} \in\{0,1\}^{m}$ for $j=0,1, \ldots, k$ such that:

(i) $G\left(m, \varepsilon_{j}\right) \subseteq G\left(n, \delta_{j}\right)$ for all $j=0,1, \ldots, k$;

(ii) $G\left(m, \varepsilon_{j}\right) \cap M=\emptyset$ for all $j=0,1, \ldots, k$;

(iii) $\operatorname{supp}\left(\varepsilon_{i}\right) \cap \operatorname{supp}\left(\varepsilon_{j}\right) \subseteq[0, n)$ whenever $0 \leq i<j \leq k$.

Proof. The proof is by induction on $k$. For $k=0$, the statement follows immediately from Lemma 4.8 (applied to $\delta=\delta_{0}$ and $l=n$ ). Assume now that the statement has been proved for some $k$. Let $\delta_{j} \in\{0,1\}^{n}$ for $j=$ $0,1, \ldots, k+1$ be given. By the induction hypothesis there exists an integer $m_{k} \geq n$ and elements $\varepsilon_{j} \in\{0,1\}^{m_{k}}$, for $j=0, \ldots, k$, such that (i), (ii) and (iii) are satisfied (with $m=m_{k}$ ). Now apply Lemma 4.8 to $\delta=\delta_{k+1}$ and $l=m_{k}$. Then there exists an integer $m_{k+1} \geq m_{k}$ and an element $\varepsilon_{k+1} \in\{0,1\}^{m_{k+1}}$ such that $\chi_{m_{k}} \varepsilon_{k+1}=\delta_{k+1}$ and $G\left(m_{k+1}, \varepsilon_{k+1}\right) \cap M=\emptyset$. Note that $\chi_{n} \varepsilon_{k+1}=\delta_{k+1}$.

It is now clear that (i) and (ii) are satisfied (with $m=m_{k+1}$ ). Since $\operatorname{supp}\left(\varepsilon_{k+1}\right) \subseteq[0, n) \cup\left[m_{k}, m_{k+1}\right]$ and $\operatorname{supp}\left(\varepsilon_{j}\right) \subseteq\left[0, m_{k}\right)$, it follows that (iii) is satisfied as well.

Before formulating the next lemma we recall that, for $k \in \mathbb{N}$ and for any $\tau \in\{0,1\}^{k+1}$, we denote by $\tau_{[0, k)} \in\{0,1\}^{k}$ the restriction of $\tau$ to $[0, k)$.

LEMMA 4.10. Let $\left\{M_{k}\right\}_{k=0}^{\infty}$ be a sequence of closed, nowhere dense subsets of $\Omega$ and let $n_{0} \in \mathbb{N} \backslash\{0\}$ and $\delta_{\emptyset} \in\{0,1\}^{n_{0}}$ be given. Then there exists a sequence $n_{0}<n_{1}<\cdots$ in $\mathbb{N}$ with the property that, for each $k \in \mathbb{N}$ and every $\tau \in\{0,1\}^{k+1}$, there exists $\delta_{\tau} \in\{0,1\}^{n_{k+1}}$ such that:

(i) $\delta_{\tau} \chi_{n_{k}}=\delta_{\tau_{[0, k)}}$ for all $\tau \in\{0,1\}^{k+1}$;

(ii) $\operatorname{supp}\left(\delta_{\tau_{1}}\right) \cap \operatorname{supp}\left(\delta_{\tau_{2}}\right) \subseteq\left[0, n_{k}\right)$ whenever $\tau_{1} \neq \tau_{2}$ in $\{0,1\}^{k+1}$;

(iii) $G\left(n_{k+1}, \delta_{\tau}\right) \cap M_{k}=\emptyset$ for all $\tau \in\{0,1\}^{k+1}$.

Proof. Suppose that $n_{0}<n_{1}<\cdots<n_{k}$ have been constructed with corresponding $\delta_{\tau} \in\{0,1\}^{n_{j}}$ for every $\tau \in\{0,1\}^{j}$ and every $j=0,1, \ldots, k$ satisfying (i), (ii) and (iii). Choose any $\tau \in\{0,1\}^{k+1}$, in which case $\tau_{[0, k)} \in$ $\{0,1\}^{k}$, and so we can define $\alpha_{\tau}=\delta_{\tau_{[0, k)}}$. Now apply Lemma 4.9 to $M=M_{k}$, $n=n_{k}$ and all the $\alpha_{\tau} \in\{0,1\}^{n_{k}}$. It follows that there exists an integer 
$n_{k+1}>n_{k}$ and elements $\delta_{\tau} \in\{0,1\}^{n_{k+1}}$ (for each $\tau \in\{0,1\}^{k+1}$ ) such that $\delta_{\tau} \chi_{n_{k}}=\alpha_{\tau}=\delta_{\tau_{[0, k)}}$ with $G\left(n_{k+1}, \delta_{\tau}\right) \cap M_{k}=\emptyset$ for all $\tau \in\{0,1\}^{k+1}$, and $\operatorname{supp}\left(\delta_{\tau_{1}}\right) \cap \operatorname{supp}\left(\delta_{\tau_{2}}\right) \subseteq\left[0, n_{k}\right)$ whenever $\tau_{1} \neq \tau_{2}$ in $\{0,1\}^{k+1}$.

Before we prove Proposition 4.7, recall that a subset $F \subseteq \Omega$ has the Baire property if there exists an open set $V \subseteq \Omega$ such that $F \triangle V$ is meager. Since the collection of all subsets with the Baire property is a $\sigma$-algebra, it is clear that any Borel subset of $\Omega$ has the Baire property.

Proof of Proposition 4.7. Let $E \in \mathcal{B}$ be non-meager in $\Omega$. It suffices to show that there exists $\mu \in \mathcal{A}$ with $\mu(E)>0$, as then $E \notin \mathcal{J}$. Since the set $E$ has the Baire property and since the sets (6) form a base for the topology in $\Omega$, it follows that there exist $n_{0} \in \mathbb{N} \backslash\{0\}$ and $\delta_{\emptyset} \in\{0,1\}^{n_{0}}$ such that $G\left(n_{0}, \delta_{\emptyset}\right) \backslash E$ is meager. Let $\left\{M_{k}\right\}_{k=0}^{\infty}$ be a sequence of closed, nowhere dense sets in $\Omega$ such that

$$
G\left(n_{0}, \delta_{\emptyset}\right) \backslash E \subseteq \bigcup_{k=0}^{\infty} M_{k} .
$$

Apply Lemma 4.10 to obtain a sequence $n_{0}<n_{1}<\cdots$ in $\mathbb{N}$ such that, for each $k \in \mathbb{N}$ and every $\tau \in\{0,1\}^{k+1}$, there exists $\delta_{\tau} \in\{0,1\}^{n_{k+1}}$ satisfying properties (i), (ii) and (iii) of the lemma.

For each $\xi \in \Omega$ define $d(\xi)=\bigcup_{k \in \mathbb{N}} \operatorname{supp}\left(\delta_{\xi_{[0, k)}}\right)$ and a function $f: \Omega \rightarrow \Omega$ by $f(\xi)=\chi_{d(\xi)}$. It follows from (i) of Lemma 4.10 that for each $k \in \mathbb{N}$,

$$
f(\xi)(n)=\delta_{\xi_{[0, k)}}(n), \quad n \in\left[0, n_{k}\right) .
$$

In particular, if $\xi, \eta \in \Omega$ satisfy $\xi_{[0, k)}=\eta_{[0, k)}$ for some $k \in \mathbb{N} \backslash\{0\}$, then $f(\xi) \chi_{n_{k}}=f(\eta) \chi_{n_{k}}$. This implies that $f$ is continuous.

Choose $\xi \in \Omega$. It follows from (8) that

$$
f(\xi) \in G\left(n_{k}, \delta_{\xi_{[0, k)}}\right) \subseteq G\left(n_{0}, \delta_{\emptyset}\right), \quad k \in \mathbb{N} \backslash\{0\} .
$$

Since $G\left(n_{k}, \delta_{\xi_{[0, k)}}\right) \cap M_{k-1}=\emptyset$ for all $k \in \mathbb{N} \backslash\{0\}$ (cf. (iii) of Lemma 4.10), this implies that $f(\xi) \notin \bigcup_{k=0}^{\infty} M_{k}$. It now follows from (7) and (9) that $f(\xi) \in G\left(n_{0}, \delta_{\emptyset}\right) \cap E \subseteq E$. Accordingly,

$$
f(\Omega) \subseteq E .
$$

Let $\lambda$ be Haar measure on $\Omega$ as given by (5) and define the probability measure $\mu=\lambda f^{-1}$, that is, $\mu(A)=\lambda\left(f^{-1}(A)\right)$ for each $A \in \mathcal{B}$. Then (10) implies that $\mu(E)=1$. It follows from the definitions of $D_{n}$ and $f$ that

$$
\mu\left(D_{n}\right)=\lambda(\{\xi \in \Omega: n \in d(\xi)\}), \quad n \in \mathbb{N} .
$$

Fix $k \in \mathbb{N}$ and assume that $n \in\left[n_{k}, n_{k+1}\right)$. It follows from (i) in Lemma 4.10 that $n \in d(\xi)$ if and only if $n \in \operatorname{supp}\left(\delta_{\left.\xi_{[0, k+1}\right)}\right)$, and hence,

$$
\mu\left(D_{n}\right)=\lambda\left(\left\{\xi \in \Omega: n \in \operatorname{supp}\left(\delta_{\left.\xi_{[0, k+1}\right)}\right)\right\}\right), \quad n \in \mathbb{N} .
$$


By Lemma 4.10(ii) there is at most one $\tau \in\{0,1\}^{k+1}$ such that $n \in \operatorname{supp}\left(\delta_{\tau}\right)$. If no such $\tau$ exists, then $\mu\left(D_{n}\right)=0$. If there exists $\tau_{0} \in\{0,1\}^{k+1}$ such that $n \in \operatorname{supp}\left(\delta_{\tau_{0}}\right)$, then

$$
\left\{\xi \in \Omega: n \in \operatorname{supp}\left(\delta_{\xi_{[0, k+1)}}\right)\right\}=\left\{\xi \in \Omega: \xi_{[0, k+1)}=\tau_{0}\right\}
$$

and so $\mu\left(D_{n}\right)=2^{-(k+1)}$. Hence, $\mu\left(D_{n}\right) \leq 2^{-(k+1)}$ for all $n \in\left[n_{k}, n_{k+1}\right)$. Accordingly, $\lim _{n \rightarrow \infty} \mu\left(D_{n}\right)=0$ and so $\mu \in \mathcal{A}$. Since $\mu(E)>0$, the proof of Proposition 4.7 is complete. In particular, Proposition 4.6 is thereby also established.

Now it is clear how to construct an example of the type announced at the beginning of this subsection. Indeed, with $\Phi$ as defined by (4), let $X_{\Phi} \subseteq \operatorname{ca}(\mathcal{B})$ be the band generated by $\Phi$. By Proposition $4.6, \mathcal{B}$ is not sequentially $\Phi$ complete. Then Theorem 3.2 implies that the Bade $\sigma$-complete B.a. $\mathcal{M}_{\Phi}$ (notation as in Theorem 3.2) cannot be sequentially closed in $\mathcal{L}_{\mathrm{S}}\left(X_{\Phi}\right)$.

We are indebted to G. Debs for an invaluable conversation, leading in particular to the suggestion that the ideal $\mathcal{J}$ is meager; a similar question was examined in [4].

4.3. Concluding remarks. Finally, we indicate how the previous example can be used to obtain an example of a Bade $\sigma$-complete B.a. of projections in a Hilbert space which fails to be sequentially closed.

The setting is still $\Omega=\{0,1\}^{\mathbb{N}}$ and with $\Phi$ as given by (4). Define $\widetilde{\Omega}=\Omega \times \Phi$ and, for every $G \subseteq \widetilde{\Omega}$ and $\nu \in \Phi$, let $G^{\nu}=\{t \in \Omega:(t, \nu) \in G\}$. Then $\Gamma=\left\{G \subseteq \widetilde{\Omega}: G^{\nu} \in \mathcal{B}\right.$ for every $\left.\nu \in \Phi\right\}$ is a $\sigma$-algebra of subsets of $\widetilde{\Omega}$. Moreover, $\mu: \Gamma \rightarrow[0, \infty]$ defined by $\mu(G)=\sum_{\nu \in \Phi} \nu\left(G^{\nu}\right)$ for $G \in \Gamma$ is a $\sigma$ additive measure and, being decomposable, $(\widetilde{\Omega}, \Gamma, \mu)$ is a Maharam measure space (cf. [7, Section 64]). Consider now the Hilbert space $\mathcal{H}=L^{2}(\mu)$. For each $A \in \mathcal{B}$ we define $P(A) \in \mathcal{L}(\mathcal{H})$ by $P(A) f=\chi_{A \times \Phi} f$ for all $f \in \mathcal{H}$. Then $P: \mathcal{B} \rightarrow \mathcal{L}(\mathcal{H})$ is a spectral measure and $\mathcal{M}=\{P(A): A \in \mathcal{B}\}$ is a Bade $\sigma$-complete B.a. of (self-adjoint) projections in $\mathcal{H}$. Given $\nu \in \Phi$ and a function $f$ on $\widetilde{\Omega}$, define $f^{\nu}: t \mapsto f(t, \nu)$ for each $t \in \Omega$. Then $f \in$ $\mathcal{H}$ if and only if there exist countably many $\left\{\nu_{k}\right\}_{k=1}^{\infty} \subseteq \Phi$ such that each $f^{\nu_{k}} \in L^{2}\left(\nu_{k}\right)$ with $\sum_{k=1}^{\infty}\left\|f^{\nu_{k}}\right\|_{2}^{2}<\infty$ and $f^{\nu}=0$ ( $\nu$-a.e. $)$ for all other $\nu \in \Phi$. This observation, together with the fact that $\nu\left(D_{n} \triangle D_{m}\right) \rightarrow 0$ as $m, n \rightarrow \infty$ for each $\nu \in \Phi$, and a standard truncation argument, shows that $\left\|P\left(D_{n}\right) f-P\left(D_{m}\right) f\right\|_{2} \rightarrow 0$ as $m, n \rightarrow \infty$ for each $f \in \mathcal{H}$. Hence, there exists a self-adjoint projection $Q$ in $\mathcal{H}$ such that $P\left(D_{n}\right) \rightarrow Q$ in $\mathcal{L}_{\mathrm{s}}(\mathcal{H})$ as $n \rightarrow \infty$. Assuming that $Q=P(E)$ for some $E \in \mathcal{B}$, it is easy to see that this would imply $\nu\left(D_{n} \triangle E\right) \rightarrow 0$ as $n \rightarrow \infty$ for each $\nu \in \Phi$. However, from Example B we know that no such set $E \in \mathcal{B}$ exists. Consequently, $\mathcal{M}$ is not sequentially closed in $\mathcal{L}_{\mathrm{s}}(\mathcal{H})$. 
In conclusion, we remark that in the above example the space $\mathcal{H}=L^{2}(\mu)$ may be replaced by the Banach space $X=L^{p}(\mu)$ for any $1 \leq p<\infty$, and we still maintain all the features that occur in $L^{2}(\mu)$.

\section{References}

[1] W. G. Bade, On Boolean algebras of projections and algebras of operators, Trans. Amer. Math. Soc. 80 (1955), 345-359.

[2] -, A multiplicity theory for Boolean algebras of projections in Banach spaces, ibid. 92 (1959), 508-530.

[3] J. Bourgain, D. H. Fremlin and M. Talagrand, Pointwise compact sets of Bairemeasurable functions, Amer. J. Math. 100 (1978), 845-886.

[4] G. Debs et J. Saint-Raymond, Ensembles boréliens d'unicité et d'unicité au sens large, Ann. Inst. Fourier (Grenoble) 37 (1987), 217-239.

[5] J. Diestel and J. J. Uhl Jr., Vector Measures, Math. Surveys 15, Amer. Math. Soc., Providence, RI, 1977.

[6] N. Dunford and J. T. Schwartz, Linear Operators III: Spectral Operators, WileyInterscience, New York, 1971.

[7] D. H. Fremlin, Topological Riesz Spaces and Measure Theory, Cambridge Univ. Press, London, 1974.

[8] —, Measure Theory, Vol. 3, Measure Algebras, Torres Fremlin, 2002.

[9] P. Meyer-Nieberg, Banach Lattices, Springer, Berlin, 1991.

[10] J. C. Oxtoby, Measure and Category, 2nd ed., Springer, Berlin, 1987.

[11] B. de Pagter and W. J. Ricker, Boolean algebras of projections and resolutions of the identity of scalar-type spectral operators, Proc. Edinburgh Math. Soc. 40 (1997), 425-435.

[12] K. P. Parthasarathy, Probability Measures on Metric Spaces, Academic Press, New York, 1967.

[13] W. J. Ricker, Sequential closures of $\sigma$-subalgebras for a vector measure, Comment. Math. Univ. Carolin. 37 (1996), 91-97.

[14] - The sequential closedness of $\sigma$-complete Boolean algebras of projections, J. Math. Anal. Appl. 208 (1997), 364-371.

[15] H. L. Royden, Real Analysis, 3rd ed., Macmillan, New York, 1988.

[16] R. Sikorski, Boolean Algebras, 3rd ed., Ergeb. Math. Grenzgeb. 25, Springer, New York, 1969.

Department of Mathematics

University of Essex

Wivenhoe Park

Colchester CO4 3SQ, United Kingdom

E-mail: fremdh@essex.ac.uk

Mathematisch-Geographische Fakultät

Katholische Universität Eichstätt-Ingolstadt

D-85071 Eichstätt, Germany

E-mail: werner.ricker@ku-eichstaett.de
Department of Applied Mathematical Analysis

Faculty EEMCS

Delft University of Technology

Mekelweg 4

2628CD Delft, The Netherlands

E-mail: b.depagter@ewi.tudelft.nl 